Journal Club

Editor's Note: These short reviews of recent JNeurosci articles, written exclusively by students or postdoctoral fellows, summarize the important findings of the paper and provide additional insight and commentary. If the authors of the highlighted article have written a response to the Journal Club, the response can be found by viewing the Journal Club at www.jneurosci.org. For more information on the format, review process, and purpose of Journal Club articles, please see https://www.jneurosci.org/content/ jneurosci-journal-club.

\title{
Understanding Commonalities and Discrepancies between Feature and Spatial Attention Effect in the Context of a Normalization Model
}

\author{
Yang Xie ${ }^{1}$ and $\mathbb{C}$ Zhewei Zhang ${ }^{1,2}$ \\ ${ }^{1}$ Institute of Neuroscience, Key Laboratory of Primate Neurobiology, CAS Center for Excellence in Brain Science and Intelligence Technology, Shanghai \\ Institutes for Biological Sciences, Chinese Academy of Sciences, Shanghai 200031, China, and 2University of Chinese Academy of Sciences, Beijing 100049, \\ China \\ Review of Ni and Maunsell
}

Attention is a process for selecting relevant information from complex sensory inputs for further processing. Attention can be classified into spatial attention and feature attention, the former denoting selective processing of stimuli in a specific location and the latter denoting selective processing of stimuli having a particular sensory feature. Many studies have demonstrated that both spatial and feature attention modulate neuronal responses and improve the signal-to-noise ratio of the encoding of the stimulus being attended. It has also been reported that both types of attention increase the magnitude of neuronal responses in multiple visual areas (Carrasco, 2011; Maunsell, 2015). Furthermore, both spatial and feature attention enhance the representation of the attended stimulus by reducing neuronal response variability (often measured with the Fano factor) and pairwise noise correlation (Cohen and Maunsell, 2009, 2011; Mitchell et al., 2009). Commonalities between spatial and feature

\footnotetext{
Received Aug. 30, 2019; revised Dec. 4, 2019; accepted Dec. 5, 2019. We thank Tianming Yang and Zhongqiao Lin for helpful discussions and comments on the paper.

The authors declare no competing financial interests.

Correspondence should be addressed to Zhewei Zhang at zwzhang@ion.ac.cn.

https://doi.org/10.1523/JNEUROSCI.2161-19.2019

Copyright $\odot 2020$ the authors
}

attention suggest that they may share a common top-down source.

The effects of attention on neuronal activity are well captured by attentional normalization models (Lee and Maunsell, 2009; Reynolds and Heeger, 2009). Normalization has been suggested as a canonical neural computation and has successfully explained many nonlinear properties of neurons (Carandini and Heeger, 2012). In the normalization model, a neuron not only is excited when a stimulus appears in its receptive field, but also is inhibited by neurons in a local network. Attention modulates the neuron's response by adjusting the amount of the feedforward excitatory drive associated with the sensory stimulus and suppressive drive from the local network.

Although the commonalities between feature and spatial attention have been widely observed, many differences have also been reported. One significant difference is that spatial attention is limited to a specific location of the retinotopic map (Womelsdorf et al., 2006), whereas feature attention affects the processing across the entire visual field (Saenz et al., 2002). Also, the temporal dynamics of the modulation of sensory neurons by the two types of attention are different (Hayden and Gallant, 2005). These observations raise several questions. How are spatial and feature attention similar in some aspects, and different in others? Can normalization explain both commonalities and discrepancies? If it cannot, what neural mechanism could explain commonalities and discrepancies between spatial and feature attention?

To address these questions, $\mathrm{Ni}$ and Maunsell (2019) trained monkeys with a direction-change detection task while recording from the medial temporal cortex (MT). In the task, at most three drifting Gabor patches were presented on a computer screen; two of them were in the receptive field of the recorded neuron, and the other was on the opposite side of the visual field. Each Gabor patch flashed every $200 \mathrm{~ms}$; then after a blank interstimulus period, its direction or contrast might change. A cue presented before Gaborpatch onset indicated which of the three locations the monkeys should attend. The monkeys were required to detect when the direction of the Gabor patch changed $<90^{\circ}$ and were rewarded for saccading to the target Gabor within 100-600 ms after the change happened. All other changes, such as direction changes $>90^{\circ}$ and changes of contrast, should be ignored.

By manipulating the direction of the Gabors and the attended location, $\mathrm{Ni}$ and Maunsell (2019) created three different task 
variants to measure the neuronal modulation elicited by normalization, spatial attention, and feature attention. To quantify the normalization effect, there were either one or two stimuli in the receptive field, moving in either the preferred or the opposite direction, and attention was guided to the Gabor outside of the receptive field, which moved orthogonally to the preferred direction. In the spatial attention task variant, the monkeys were cued to attend to a Gabor in the receptive field, whose direction kept changing so that no specific feature was attended and the effect of feature attention was minimized. In the feature attention variant, attention was cued and attracted to the Gabor outside of the receptive field to remove the effect of spatial attention on the recorded neuron. The attended Gabor moved in the neuron's preferred direction and the moving direction was the attended feature.

The authors measured the effect of normalization, spatial attention, and feature attention on MT neurons' responses with normalization modulation index (NMI), spatial modulation index (SMI) and feature modulation index (FMI), respectively. Results showed that SMIs and FMIs were correlated across neurons, and the correlation existed even when controlling for NMIs. The authors attributed the correlation between FMIs and SMIs to the commonality between the mechanism supporting spatial and feature attention. However, as previous researches have shown, attentional modulation strength was also correlated with behavioral performance features like accuracy and reaction time (Cook and Maunsell, 2002; Cohen and Maunsell, 2011). Because the data in this experiment were collected from different neurons on different days, it was possible that the SMI/FMI correlation across neurons simply reflected the behavioral fluctuation across days. To exclude this possibility, one could calculate the SMI/FMI correlation while controlling for behavior performance. Simultaneous recordings in the same session can also address this potential confounder.

Prior studies had shown that the variance of spatial-attention modulation strength across neurons could be explained largely by the variance in normalization strength ( $\mathrm{Ni}$ et al., 2012; Verhoef and Maunsell, 2016). Ni and Maunsell (2019) tested whether feature-attention modulation strength was correlated with normalization strength and found no correlation, indicating that normalization might explain the difference between spatial and feature attention.

To better understand commonalities and discrepancies between spatial and fea- ture attention, the authors fitted the electrophysical results with a spatially-tuned equal maximum suppression normalization model they proposed previously ( $\mathrm{Ni}$ and Maunsell, 2017). In the model, a neuron's response was described as a sum of excitatory drives associated with stimuli appearing in the RF and each of them was divided by a suppressive drive separately before summation. Further, the suppressive drive changed with the associated stimulus' location, despite the stimulus always being in the RF. The authors assumed that spatial and feature attention enhanced the excitatory drive associated with the attended stimulus. Because the experimental results indicated that only spatial attention interacted with local normalization, the authors hypothesized that spatial attention modified the suppressive drive of the attended stimulus and feature attention did not. This model successfully explained variance in the neuronal responses, which suggests spatial and feature attention have the same top-down mechanism but differ in their interactions with localized normalization.

It is not clear what the biological mechanism underlying normalization is, even though many models and hypotheses have been proposed (Carandini and Heeger, 2012). A single neural mechanism cannot explain normalization across all brain areas and species. Normalization can be implemented not only through lateral inhibitory inputs from nearby neurons, but also through feedforward inputs or feedback inputs from higher brain areas. Most of these hypotheses assume that there is one unified suppressive drive and excitatory drives are summed before normalization. It is unknown whether these models could extend and reproduce the neuronal response patterns predicted by the equal maximum suppression normalization model proposed by $\mathrm{Ni}$ and Maunsell (2019), in which nonuniform suppressive drives are necessary and excitatory drives are summed after normalization. One possibility is that excitatory drives are normalized by suppressive drives before MT and then summed together. In other words, a pair of neurons with overlapping receptive fields from primary sensory areas project to the same neuron in MT and normalization happens both in primary sensory areas and MT.

Attention modulates not only the magnitude of neuronal responses, but also the noise correlation. Many factors may contribute to the noise correlation, such as local connectivity and global fluctuation caused by top-down inputs (Kohn et al., 2016). A previous study suggested that the effect of attention on the noise correlation could also be captured by a normalization model (Verhoef and Maunsell, 2017). However, in Verhoef and Maunsell's (2017)study, limited by the behavioral paradigm, different types of attention modulation could not be disentangled. With the paradigm designed by $\mathrm{Ni}$ and Maunsell (2019), researchers could investigate how spatial and feature attention exert leverage on the noise correlation individually. It would provide additional evidence toward the common source hypothesis if discrepancies between spatial and feature attention modulation on the noise correlation could be mostly explained by the normalization mechanism.

Contrary to the work by Ni and Maunsell (2019), which supports the hypothesis that spatial and feature attention have the same top-down mechanism, a study by Bichot et al. (2015) showed that a lesion in the ventral pre-arcuate region of prefrontal cortex impaired feature attention signals in the frontal eye fields but left spatial attention signals intact. One possibility that might reconcile these seemingly contrary results is that the SMI/FMI correlation in MT neurons reflects intrinsic properties of the connections between MT neurons and neurons in the brain areas that are involved in the transmission of both spatial and feature attention signals. MT neurons that are strongly connected with the brain areas, such as the frontal eye fields, conveying both types of attention signals may show relatively large SMIs and FMIs. In comparison, MT neurons that are weakly connected with the brain areas conveying both types of attention signals may show small SMIs and FMIs. Consequently, we could observe the SMI/FMI correlation even if the ventral pre-arcuate region of prefrontal cortex is only causally related to feature attention. Further investigation is needed to examine this possibility.

With a well designed behavioral task, $\mathrm{Ni}$ and Maunsell (2019) studied normalization, spatial attention, and feature attention in a unified framework that helps us to understand how top-down attention interacts with local normalization. Unlike in previous studies, when measuring the attentional effects, $\mathrm{Ni}$ and Maunsell (2019) regarded the neuronal responses in the normalization task variant as a common baseline, which allowed a reliable comparison between spatial and feature attention. Finally, Ni and Maunsell (2019) is one of the few studies comparing spatial attention and feature attention in a quan- 
titative manner (Cohen and Maunsell, 2011), which provides further clues on the relationship between mechanisms underlying spatial and feature attention.

\section{References}

Bichot NP, Heard MT, DeGennaro EM, Desimone R (2015) A source for feature-based attention in the prefrontal cortex. Neuron $88: 832-844$

Carandini M, Heeger DJ (2012) Normalization as a canonical neural computation. Nat Rev Neurosci 13:51-62.

Carrasco M (2011) Visual attention: the past 25 years. Vision Res 51:1484-1525.

Cohen MR, Maunsell JH (2009) Attention improves performance primarily by reducing interneuronal correlations. Nat Neurosci 12:1594-1600.

Cohen MR, Maunsell JH (2011) Using neuronal populations to study the mechanisms underlying spatial and feature attention. Neuron 70:1192-1204.
Cook EP, Maunsell JH (2002) Attentional modulation of behavioral performance and neuronal responses in middle temporal and ventral intraparietal areas of macaque monkey. J Neurosci 22:1994-2004.

Hayden BY, Gallant JL (2005) Time course of attention reveals different mechanisms for spatial and feature-based attention in area V4. Neuron 47:637-643.

Kohn A, Coen-Cagli R, Kanitscheider I, Pouget A (2016) Correlations and neuronal population information. Annu Rev Neurosci 39: 237-256.

Lee J, Maunsell JH (2009) A normalization model of attentional modulation of single unit responses. PLoS One 4:e4651.

Maunsell JHR (2015) Neuronal mechanisms of visual attention. Annu Rev Vis Sci 1:373-391.

Mitchell JF, Sundberg KA, Reynolds JH (2009) Spatial attention decorrelates intrinsic activity fluctuations in macaque area V4. Neuron 63:879-888.

Ni AM, Maunsell JH (2017) Spatially tuned normalization explains attention modulation variance within neurons. J Neurophysiol 118: 1903-1913.

Ni AM, Maunsell JH (2019) Neuronal effects of spatial and feature attention differ due to normalization. J Neurosci 39:5493-5505.

Ni AM, Ray S, Maunsell JH (2012) Tuned normalization explains the size of attention modulations. Neuron 73:803-813.

Reynolds JH, Heeger DJ (2009) The normalization model of attention. Neuron 61:168-185.

Saenz M, Buracas GT, Boynton GM (2002) Global effects of feature-based attention in human visual cortex. Nat Neurosci 5:631-632.

Verhoef BE, Maunsell JH (2016) Attention operates uniformly throughout the classical receptive field and the surround. eLife 5:e17256.

Verhoef BE, Maunsell JH (2017) Attentionrelated changes in correlated neuronal activity arise from normalization mechanisms. Nat Neurosci 20:969-977.

Womelsdorf T, Anton-Erxleben K, Pieper F, Treue S (2006) Dynamic shifts of visual receptive fields in cortical area MT by spatial attention. Nat Neurosci 9:1156-1160. 